\title{
The implementation of "Intranet based therapeutic schemata" for comprehensive diagnostic and therapy guidelines
}

\author{
Astrid E Sonnleitner ${ }^{1 *}$, Gerald Wendelin ${ }^{1}$, Jörg Jahnel ${ }^{2}$, Sebastian Bauchinger ${ }^{1}$, Elisabeth Thierrichter ${ }^{1}$, \\ Sabine Löffler ${ }^{1}$ \\ From Safety in hospitals: from strategy to implementation Annual Scientific Meeting 2015 \\ Graz, Austria. 29-30 September 2015
}

\section{Background}

In former times in many pediatric fields no easily accessible standardized and regularly reviewed guidelines for various medical patterns were available in the Department of Pediatrics and Adolescent Medicine of the Medical University of Graz.

\section{Material and methods}

To avoid out-of-date diagnostic and unstandardized treatment in the Department of Pediatrics and Adolescent Medicine and to provide a common workup regarding diagnostic pathways and treatment - regardless of the level of medical education of clinicians - an internet database called "Intranet based therapeutic schemata" (ITS) for a standardized diagnostic and treatment concept was set up.

Clinicians, each of them experts in different pediatric fields, were assigned to elaborate common concepts regarding diagnostic workup and therapy pathways. These files were implemented in this newly generated internet database ITS and are accessible on every computer in the Department of Pediatrics and Adolescent Medicine.

Key Users monitor these files on a regular basis and tag each file with a color label (red, yellow, green), indicating the up-to-dateness. A green label indicates a review within the last 3 years, a yellow one within 3 to 6 years and a red label implies that no review has been done within the last 6 years.

In course of this process authors of files with a red label are asked to review their guidelines. If a red

\footnotetext{
* Correspondence: astrid.sonnleitner@medunigraz.at

'Department of Pediatrics and Adolescent Medicine, Medical University of Graz, Graz, Austria

Full list of author information is available at the end of the article
}

labeled file is not reviewed within 1 year, it will be deleted by the Key Users.

To transfer new information to all clinicians in the hospital, information about the implementation of new guidelines is sent by email to every MD. In the database new files are highlighted for the duration of one month. Additionally once a year a conference is held, where new ITS topics are presented and consequently discussed.

\section{Results}

Due to this easily accessible information a common diagnostic and treatment regimen for many diseases could be implemented. Especially for new MD it is a useful tool to provide standardized diagnostic assessment and treatment. In rare clinical questions, especially legal patterns, it provides useful information for a faster approach to certain situations.

\section{Conclusions}

Further extension will be needed to cover as consistently as possible most medical questions of clinician's routine.

\section{Authors' details \\ ${ }^{1}$ Department of Pediatrics and Adolescent Medicine, Medical University of Graz, Graz, Austria. ${ }^{2}$ Department of Pediatrics and Adolescent Medicine, Division of General Pediatrics, Medical University of Graz, Graz, Austria. \\ Published: 30 October 2015 \\ doi:10.1186/2056-5917-1-S1-A26 \\ Cite this article as: Sonnleitner et al:: The implementation of "Intranet based therapeutic schemata" for comprehensive diagnostic and therapy guidelines. Safety in Health 2015 1(Suppl 1):A26.}

